\title{
Comparative analysis of gene expression profiles in differentiated subcutaneous adipocytes between Jiaxing Black and Large White pigs
}

Dawei Zhang ${ }^{1 \dagger}$, Wenjing $\mathrm{Wu}^{1 \dagger}$, Xin Huang ${ }^{2}, \mathrm{Ke} \mathrm{Xu}^{2}$, Cheng Zheng ${ }^{2}$ and Jin Zhang ${ }^{1 *}$

\begin{abstract}
Background: Chinese domestic pig breeds are reputed for pork quality, but their low ratio of lean-to-fat carcass weight decreases production efficiency. A better understanding of the genetic regulation network of subcutaneous fat tissue is necessary for the rational selection of Chinese domestic pig breeds. In the present study, subcutaneous adipocytes were isolated from Jiaxing Black pigs a Chinese indigenous pig breed with redundant subcutaneous fat deposition and Large White pigs a lean-type pig breed with relatively low subcutaneous fat deposition. The expression profiles of mRNAs and IncRNAs were compared by RNA-seq analysis to identify biomarkers correlated with the differences of subcutaneous fat deposition between the two breeds.

Results: A total of 1058 differentially expressed genes and 221 differentially expressed IncRNAs were identified in subcutaneous adipocytes between Jiaxing Black and Large White pigs, which included 275 up-regulated mRNAs, 783 down-regulated mRNAs, 118 up-regulated IncRNAs and 103 down-regulated IncRNAs. Gene Ontology and KEGG pathway enrichment analyses revealed that the differentially expressed genes and differentially expressed IncRNAs were mainly involved in the immune response, cell fate determination, PI3K-Akt signaling pathway and MAPK signaling pathway, which are known to be related to adipogenesis and lipid metabolism. The expression levels of differentially expressed genes and differentially expressed IncRNAs according to the RNA-seq data were verified by quantitative PCR, which showed $81.8 \%$ consistency. The differences in MAPK pathway activity between Jiaxing Black and Large White pigs was confirmed by western blot analysis, which revealed elevated p38 phosphorylation in Jiaxing Black pigs.
\end{abstract}

Conclusions: This study offers a detailed characterization of mRNAs and IncRNAs in fat- and lean-type pig breeds. The activity of the MAPK signaling pathway was found to be associated with subcutaneous adipogenesis. These results provide new targets for further investigation of the molecular mechanisms regulating subcutaneous fat deposition in pigs.

Keywords: Subcutaneous fat, Pig, Sus scrofa, LncRNA, mRNA, RNA-seq

\footnotetext{
* Correspondence: zhangjin7688@163.com

${ }^{\dagger}$ Dawei Zhang and Wenjing Wu contributed equally to this work.

'College of Biological, Chemical Science and Engineering, Jiaxing University, Jiaxing 314001, China

Full list of author information is available at the end of the article
}

(c) The Author(s). 2021 Open Access This article is licensed under a Creative Commons Attribution 4.0 International License, which permits use, sharing, adaptation, distribution and reproduction in any medium or format, as long as you give appropriate credit to the original author(s) and the source, provide a link to the Creative Commons licence, and indicate if changes were made. The images or other third party material in this article are included in the article's Creative Commons licence, unless indicated otherwise in a credit line to the material. If material is not included in the article's Creative Commons licence and your intended use is not permitted by statutory regulation or exceeds the permitted use, you will need to obtain permission directly from the copyright holder. To view a copy of this licence, visit http://creativecommons.org/licenses/by/4.0/. The Creative Commons Public Domain Dedication waiver (http://creativecommons.org/publicdomain/zero/1.0/) applies to the data made available in this article, unless otherwise stated in a credit line to the data. 


\section{Background}

Fat deposition is one of the most important economic traits of pigs. The amount of subcutaneous (SC) fat deposits is associated with lean meat carcass percentage, while intramuscular (IM) fat content is the main factor affecting pork quality [1]. Foreign pig breeds, such as Duroc, Large White (LW), and Landrace, deposit low levels of SC fat, while Chinese indigenous pig breeds, such as Laiwu, Taihu, and Jinhua, deposit high levels of SC fat [2-6]. Excessive SC fat deposition greatly decreases the growth performance and meat production efficiency, which results in profit reduction [7, 8]. However, Chinese indigenous pig breeds often exhibit better fertility, disease resistance and IM fat content than foreign pig breeds $[9,10]$. Understanding the porcine adipocyte regulation network to decrease SC fat deposition is a key issue in genetic improvement of Chinese indigenous pig breeds. In addition, human health problems caused by excessive fat accumulation are becoming increasingly common. It has been demonstrated that obesity increases the risk for the development of type 2 diabetes mellitus, cardiovascular disease, hypertension, dyslipidemia, and certain types of cancer [11, 12]. Notably, pigs can be used in biomedical studies due to their anatomic and physiological similarity to humans [13, 14]. Therefore, clarifying the molecular mechanisms of $\mathrm{SC}$ fat deposition in pigs can not only benefit the genetic breeding of pigs, but also deepen our understanding of human metabolic diseases.

Porcine SC fat deposition is largely determined by the proliferation and differentiation of adipocytes [15]. With the advent of omics technologies, many genes and pathways regulating the metabolism of porcine adipocytes have been identified [16-18]. Zhang et al. [19, 20] reported that apolipoprotein $\mathrm{R}$ is the key molecule promoting lipolysis in porcine adipocytes according to DNA microarray analysis. Wu et al. [21] demonstrated that C1q/tumor necrosis factor-related protein 6 (C1QTNF6) regulates porcine $\mathrm{SC}$ fat deposition via the MAPK and p53 signaling pathways using RNA-seq analysis. Recently, the regulatory role of long noncoding RNAs (lncRNAs) in porcine adipogenesis has garnered increasing attention $[22,23]$. LncRNAs are defined as a class of transcribed RNA molecules that are more than $200 \mathrm{nu}$ cleotides in length and do not encode proteins [24]. LncRNAs can interact with DNA, RNA or proteins, and regulate gene expression via diverse mechanisms [25]. Identifying the regulatory role of lncRNAs in porcine adipogenesis is of great importance for understanding the molecular mechanisms that regulate SC fat deposition in pigs. Although several reports on lncRNAs in porcine adipose tissue were published in the past 2 years, our understanding how lncRNAs regulate fat deposition in pigs is still very limited.
Jiaxing Black (JX) pig, a Chinese indigenous pig breed in the Taihu Lake region, is characterized by its early sexual maturity, high fecundity and crude feed tolerance. Additionally, it is renowned for the good performance of its hybrids with foreign pig breeds, and plump muscles with a high content of IM fat. Products derived from JX pigs have been developed into a well-recognized commercial pork brand in China [26]. However, the excessive SC fat deposition decreases the growth efficiency and results in profit reduction. By contrast, LW pigs are the most widely distributed lean-type pig breed with relatively low SC fat deposition [10]. In this study, highthroughput RNA-seq was conducted to compare the gene expression profiles of differentiated SC adipocytes from the two pig breeds. LncRNAs and genes associated with porcine adipogenesis or lipid metabolism were identified. Furthermore, functional enrichments and interaction network analyses were conducted to investigate the molecular mechanisms of differentially expressed lncRNAs (DELs) and genes regulating fat deposition, which provides new relevant data for understanding the regulatory network of SC fat deposition in pigs.

\section{Results}

\section{RNA-seq analysis of SC adipocytes from JX pig and LW}

\section{pig}

Primary SC adipocytes were isolated from three JX and three LW pigs (3 days old), and subjected to 8 days of differentiation. The differentiated adipocytes were harvested and subjected to RNA-seq analysis in three biological replicates. The Illumina HiSeq 2000 platform provided an average of $15.2 \mathrm{~GB}$ of clean reads for each sample. The percentage of clean reads among the raw data in each library ranged from 91.44 to $95.10 \%$. For each sample, 90.22, 87.20, 86.05, 84.79, 85.59 and $85.76 \%$ were uniquely mapped to the current version of the pig genome (Sscrofa 11.1), representing 12,446, 11, $643,11,765,11,579,11,593$ and 11,310 genes, respectively (Additional file 1: Supplementary Table 1). Gene numbers within a defined range of FPKM values (FPKM $\leq 1$, FPKM $1 \sim 10$ and FPKM $\geq 10$ ) were analyzed, and each sample gave similar results (Additional file 1: Supplementary Fig. 1A). In each breed, the abundance of mRNAs was relatively higher than that of lncRNAs, as expected, while both mRNAs and lncRNAs showed similar distribution in both breeds (Additional file 1: Supplementary Figs. $1 \mathrm{~B}$ and $1 \mathrm{C}$ ). The transcripts that met at least three of four criteria (CPC, txCdsPredict, CNCI and Pfam) were identified as candidates lncRNAs, which yielded 4165 lncRNAs for subsequent analysis (Additional file 1: Supplementary Fig. 1D). The majority of known lncRNAs have two to four exons, while the novel lncRNAs mainly had one to three exons 
(Additional file 1: Supplementary Fig. 1E). In addition, the transcript abundance of housekeeping genes such as EEF1A1, ACTA2 and GAPDH was high, as can be seen in supplementary Table 2 (Additional file 1). Taken together, both the biological replicates and sequencing data indicated sufficiently good data quality for further analysis.

\section{Differentially expressed IncRNAs and genes in SC adipocytes from the two breeds}

To further understand the differences of SC adipocytes between the two breeds, comparative transcriptome analysis was conducted, and the minimum FPKM value of gene expression was greater than or equal to 1 . A total of 1279 genes (1058 coding genes and 221 lncRNAs) were differentially expressed, including 393 up- and 886 down-regulated genes in SC adipocytes between the two breeds (Fig. 1a). Among the 1058 differentially expressed genes (DEGs), 275 were up- and 783 were downregulated, and the FPKM values of some DEGs exhibited great differences between JX and LW pigs, including KRT5, UBC, LDHB, C1QTNF3 and RAMP1 (Additional file 1: Supplementary Table 3). Among the 221 DELs, 118 were up- and 103 were down-regulated (Fig. 1b). Among these DEGs and DELs, 797 DEGs and 40 DELs had been previously annotated, and 261 DEGs and 181 DELs were novel (Fig. 1c).

\section{Functional enrichment analysis of DEGs}

The potential functions and signaling pathways of all DEGs were subjected to Gene Ontology (GO) and Kyoto Encyclopedia of Genes and Genomes (KEGG) enrichment analysis. GO analysis based on biological process was conducted and the top 20 most highly enriched categories with $P<0.05$ were listed (Fig. 2a). The results showed that DEGs related to cell differentiation,

A
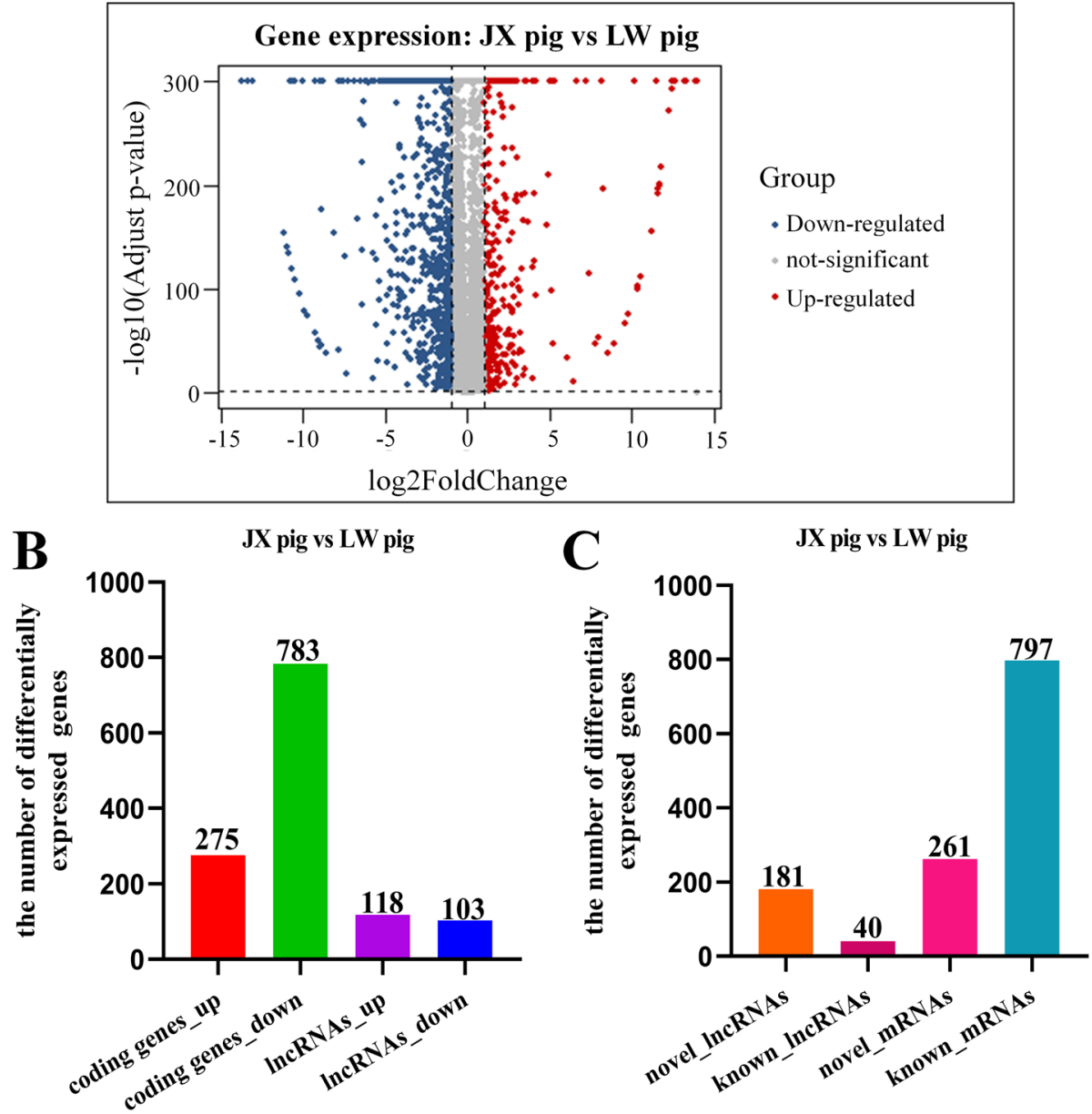

Fig. 1 Differential expression characteristics of coding genes and IncRNAs in SC adipocytes between LW and JX pigs. a The volcano plot of differentially expressed coding genes and IncRNAs. b Quantitive comparison of the up- and down-regulated genes. c The number of known and novel genes 

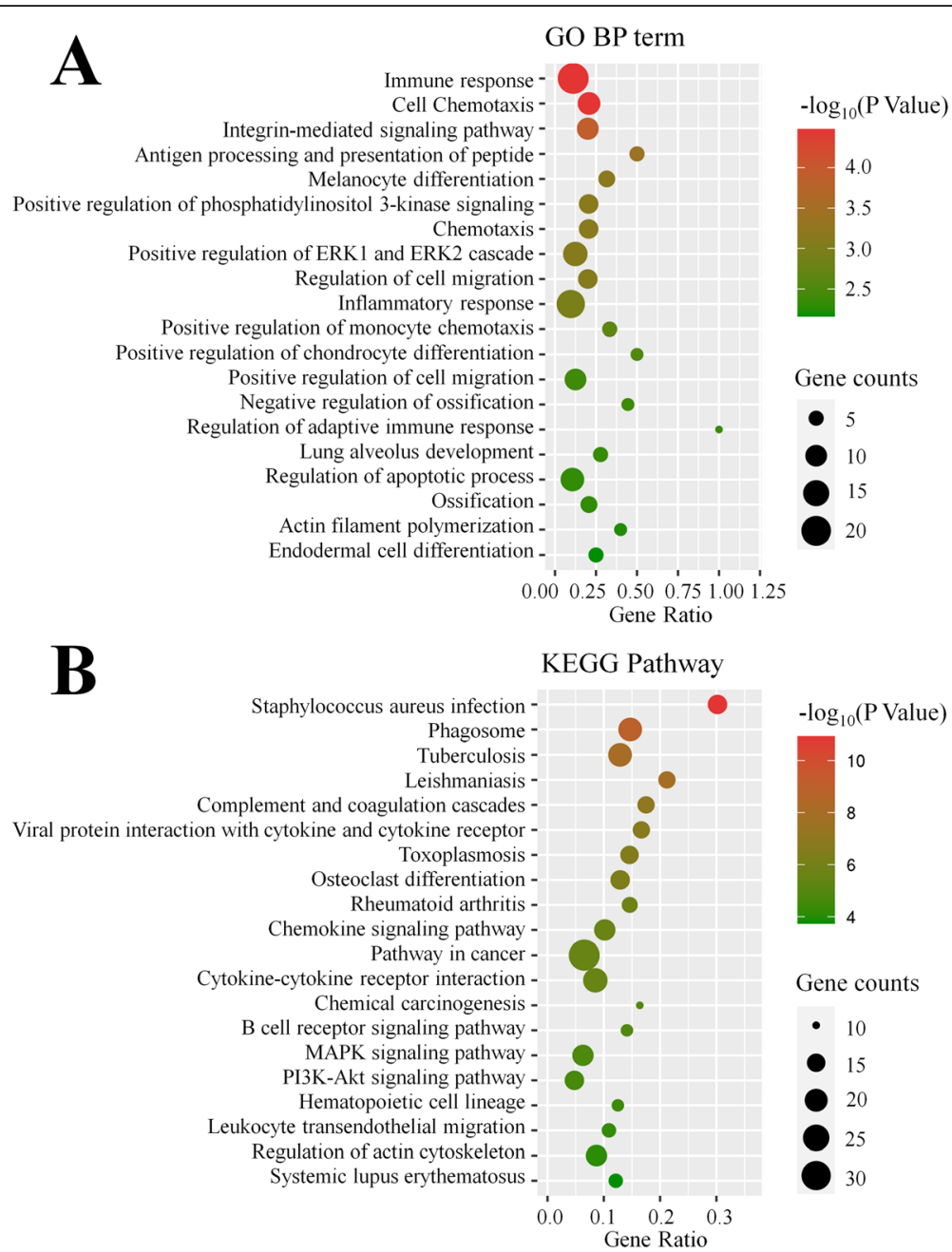

Fig. 2 GO annotation and KEGG pathway analysis of DEGs. a Go terms distribution of DEGs under biological processes. b Enrichment of DEGs in signaling pathways. Each bubble represents a term. The size of the bubble indicates the number of involved genes. The colors indicate $P$ values, and the significance level of enrichment was set at $P<0.05$, and enrichment terms was ranked by $P$ values

migration, and apoptosis were significantly enriched. Five processes related to immunity, namely "Immune response", "Antigen processing and presentation of peptide", "Inflammatory response", "Positive regulation of monocyte chemotaxis", and "Regulation of adaptive immune response", were detected. Two terms closely associated with lipid metabolism were also identified, including "Positive regulation of phosphatidylinositol 3kinase signaling" and "Positive regulation of ERK1 and ERK2 cascade". In addition, KEGG enrichment analysis was performed and the top 20 pathways are presented in Fig. 2b. Among these results, several immune-related pathways were also found, such as "Staphylococcus aureus infection", "Phagosome", "Tuberculosis", "Complement and coagulation cascades", "Viral protein interaction with cytokine and cytokine receptor", "Rheumatoid arthritis", "Chemokine signaling pathway",
"Cytokine-cytokine receptor interaction", "B cell receptor signaling pathway", "Leukocyte transendothelial migration", and "Systemic lupus erythematosus". Moreover, "Osteoclast differentiation", "MAPK signaling pathway" and "PI3K-Akt signaling pathway" were significantly enriched, all of which are highly associated with adipocyte differentiation and lipid accumulation. The results of enrichment analysis illustrated the regulatory differences of SC fat deposition between JX and LW pigs.

\section{Protein-protein interaction network analysis}

In unsupervised hierarchical clustering analysis, heat maps were generated using the DEGs, and they clearly self-segregated into different clusters for JX and LW pigs. These results reflected the distinct mRNA expression profiles of the two breeds (Fig. 3a). The proteinprotein network was constructed based on the Maximal 


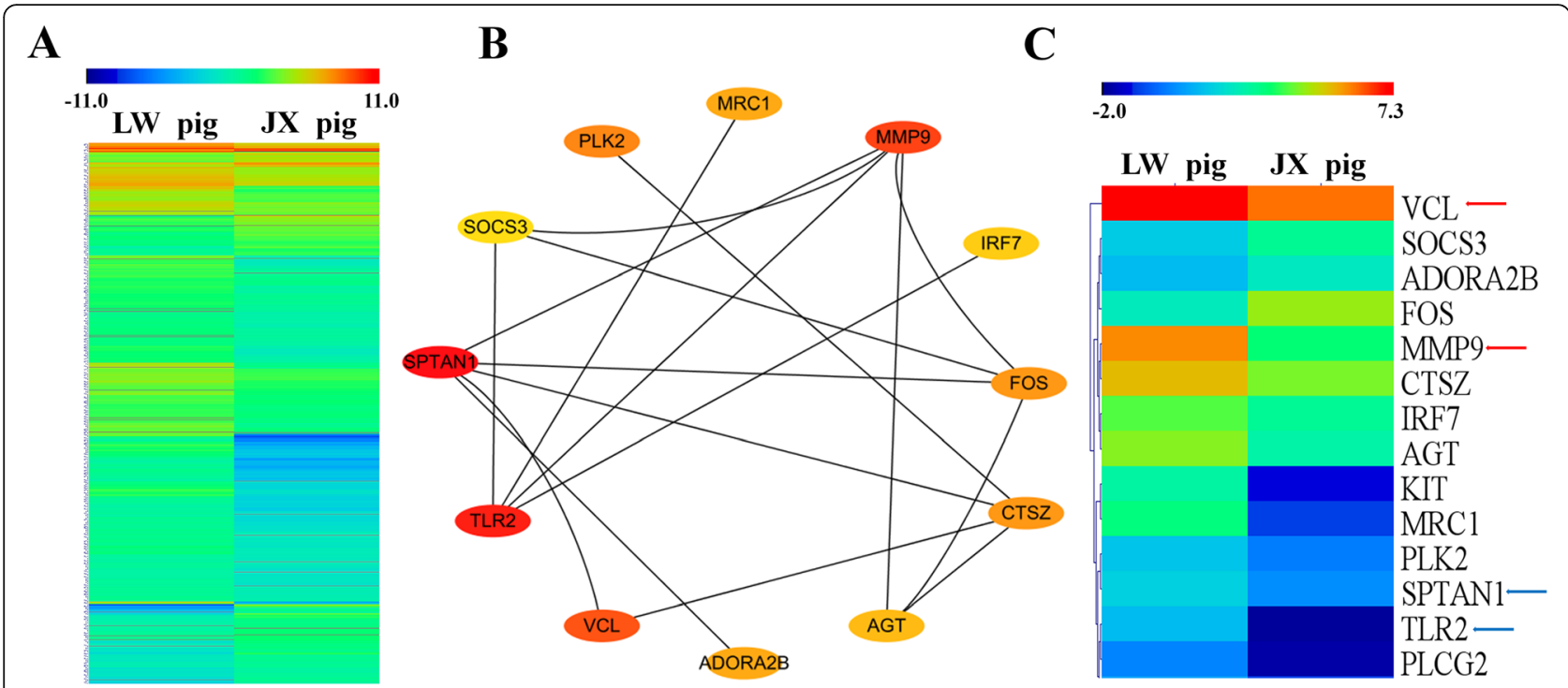

Fig. 3 Protein-protein interaction (PPI) network analysis by cytoscape of DEGs. a Unsupervised hierarchical clustering of the expression profile of significant genes of DEGs. $\mathbf{b}$ The PPI network of DEGs according to MCODE. $\mathbf{c}$ Unsupervised hierarchical clustering of the expression profile of hub genes in PPI network, and the arrows (red: up-regulated; blue: down-regulated) indicate four DEGs with high interaction scores

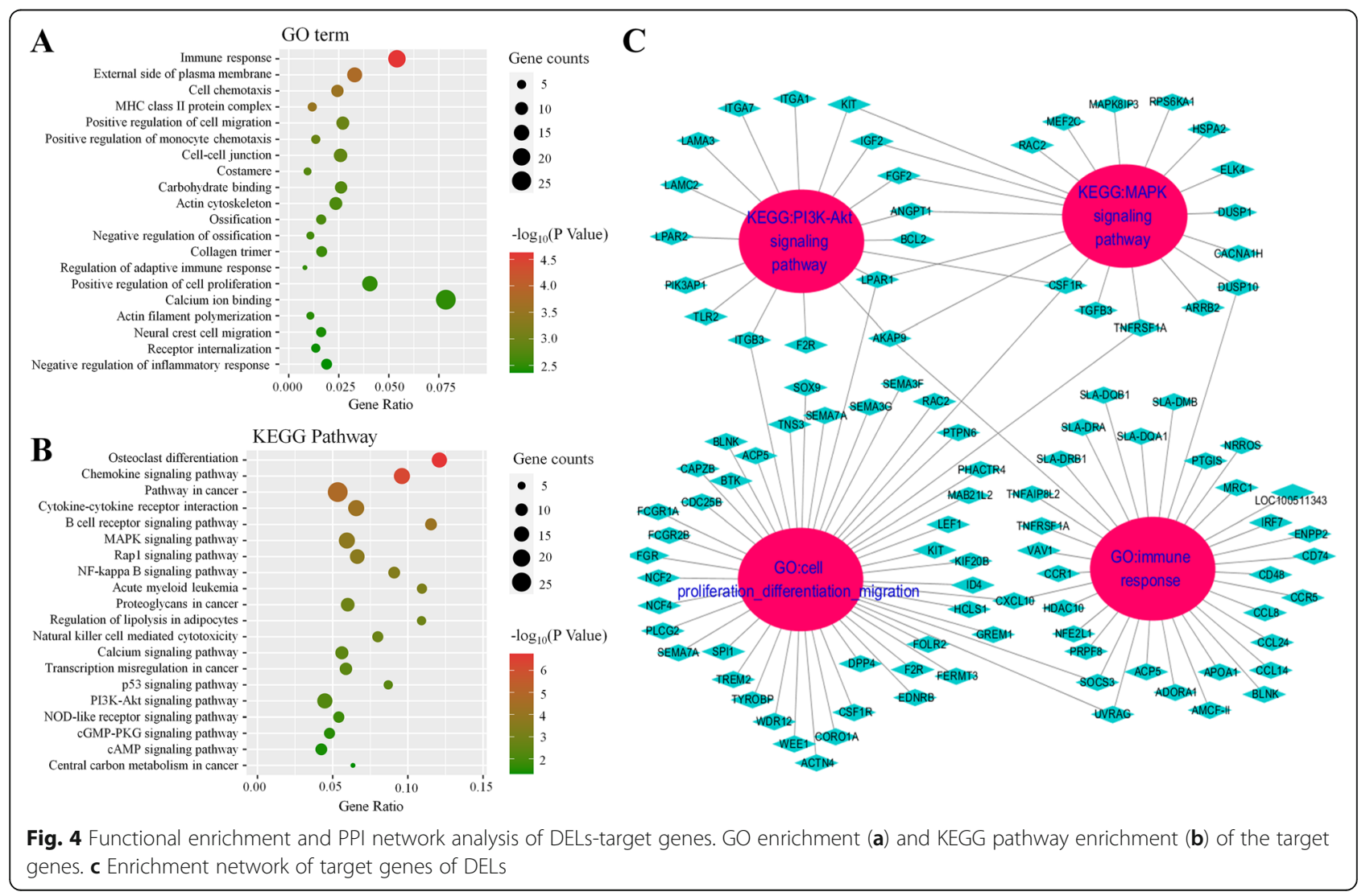


Clique Centrality (MCC) topological algorithm. According to the interaction scores, four DEGs exhibited obvious strong connections with other genes. Among the four DEGs, MMP9 and $V C L$ were up-regulated, while SPTAN1 and TLR2 were down-regulated in JX pigs compared to LW pigs (Fig. 3c).

\section{Functional enrichment analysis of IncRNAs based on target genes}

Based on the RNA-seq data, the potential target genes of DELs were predicted to explore their potential functions (Additional file 2: Supplementary Table 4). In GO enrichment analysis, the five immunity-related terms "Immune response", "MHC class II protein complex", "Positive regulation of monocyte chemotaxis", "Regulation of adaptive immune response" and "Negative regulation of inflammatory response", were detected. The categories cell chemotaxis, cell migration and ossification, which were detected in the DEG analysis, were also identified here (Fig. 4a). In KEGG enrichment analysis, seven pathways related to adipocyte differentiation and lipid accumulation were identified, including "MAPK signaling pathway", "Regulation of lipolysis in adipocyte", "Calcium signaling pathway", "p53 signaling pathway", "PI3K-Akt signaling pathway", "cGMP-PKG signaling pathway", and "cAMP signaling pathway" (Fig. 4b). In addition, the target genes enriched in four categories, including "MAPK signaling pathway", "PI3K-Akt signaling pathway", "Immune response", and "Cell proliferation, differentiation and migration" were shown in Fig. 4c, and they were also identified in the enrichment analysis of DEGs. Among the target genes, WDR12, LPAR1, WEE1, CDC25B, CAPZB and UVRAG are known to participate in the regulation of cell proliferation or differentiation. Furthermore, PRPF8, AKAP9, UVRAG, HDAC10 and NFE2L1 are known to mediate the immune response. Moreover, LPAR1 and AKAP9 were also reported to be associated with the PI3K/AKT signaling pathway or MAPK signaling pathway. Significantly, $N f e 2 l 1$ was reported to have an impact on the plasticity of adipose tissue. Thus, the DELs might play an essential role in the distinct adipogenesis of JX pigs.

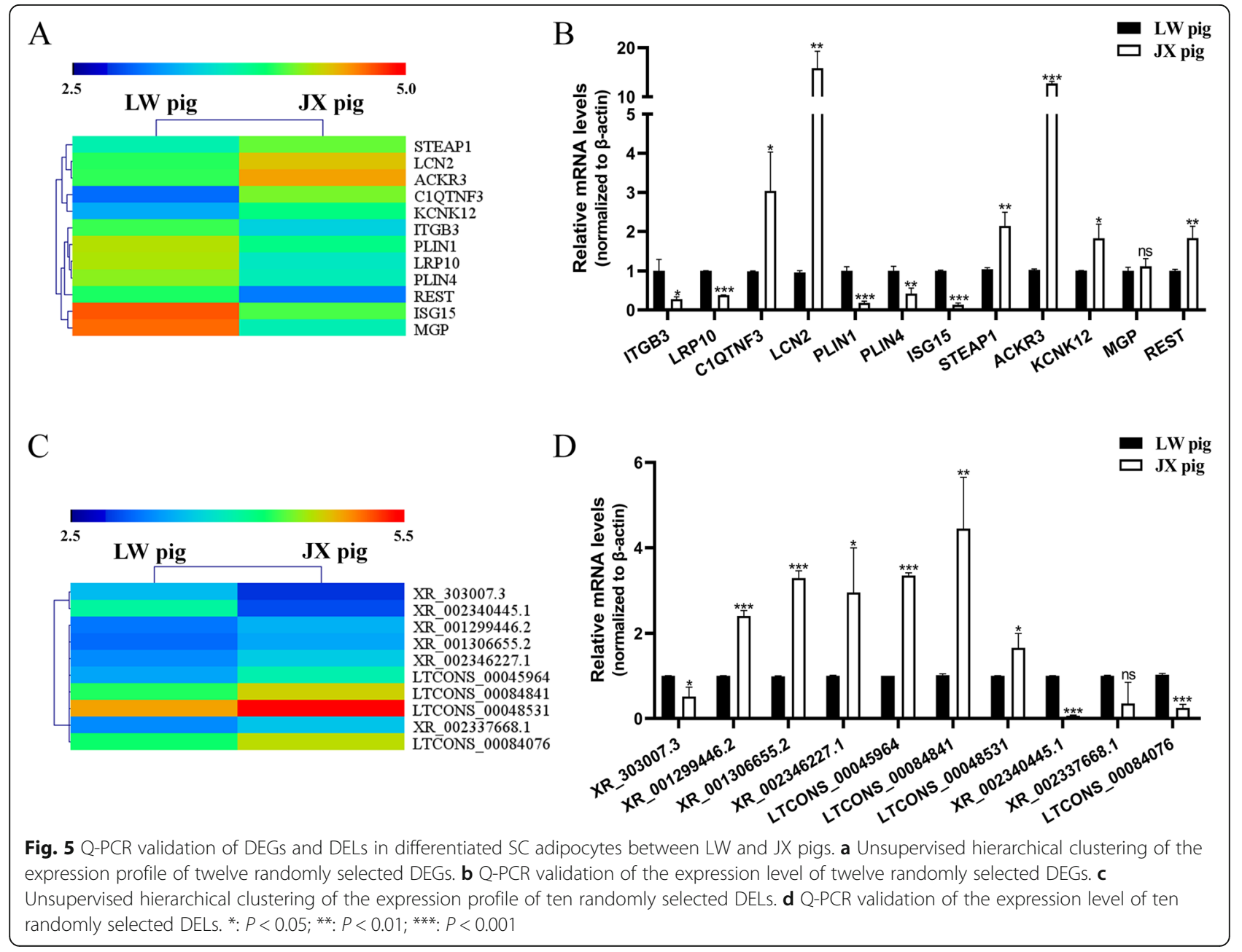


Validation of the DEGs and DELs

To validate the reliability of the RNA-seq results, 12 DEGs and 10 DELs were randomly chosen for quantitative PCR (qPCR) verification (Fig. 5a-d). Compared with the RNAseq data, 10 DEGs and 8 DELs gave consistent results, while two DEGs (MGP and RESTI) and two DELs (XR 002337668.1 and LTCONS_00084076) showed statistically different results by qPCR analysis. Overall, $81.8 \%$ of the results were in agreement between the two techniques.

\section{Verification of the pathway analysis}

Because the MAPK signaling pathway was identified in the functional enrichment analysis of both DEGs and DELs, its activity in SC fat tissues of the two breeds was examined. Expression levels and phosphorylation of two kinases in the MAPK pathway, ERK1/2 and p38, were determined by western blot analysis. ERK1/2 showed no differences in total protein abundance or phosphorylation between the two breeds. However, while the total protein abundance of p38 was similar, the abundance of phosphorylated p38 showed an obvious difference between the two breeds. Accordingly, the samples from JX pigs showed higher p38 phosphorylation levels than the samples from LW pigs (Fig. 6 and Additional file 3: Supplementary Fig. 2). The difference of p38 phosphorylation between the two breeds supported the results of pathway enrichment analysis.

\section{Discussion}

SC fat tissue has multiple functions in pigs, including thermal insulation, energy storage and adipokine secretion $[27,28]$. However, the reduction of SC fat content is of great importance for pig production because fat deposition wastes a lot of energy [7]. Therefore, excessive triglyceride accumulation in SC fat tissues is unfavorable for both energy utilization and lean meat production in pigs. Many Chinese domestic pig breeds are reputed for their high pork quality, but their low ratio of lean-to-fat carcass weight decreases production efficiency. Consequently, a better understanding of the regulation network controlling SC fat tissue deposition is necessary for the rational genetic improvement of Chinese domestic pig breeds. In this study, the gene expression profiles of SC adipocytes from the local JX pig breed and foreign LW pig breed were compared via RNA-seq analysis. A total of 1279 differentially expressed genes were identified, including 1058 coding genes (DEGs) and 221 lncRNAs (DELs). These results were validated by $\mathrm{qPCR}$ analysis, which indicated that the data are reliable, with $81.8 \%$ consistency. Interestingly, we noticed that there were more down-regulated DEGs than up-regulated DEGs. This result indicated that many genes are suppressed in the SC adipocytes of JX pigs, which may be related to vigorous adipogenesis inside the cells.

In order to identify the differences between the regulation networks of SC adipocytes from the two pig breeds, GO and KEGG pathway enrichment analyses were performed. DEGs and target genes of DELs were mainly enriched in three pathways related to lipid metabolism and adipocyte differentiation, namely "Calcium signaling pathway", "PI3K-Akt signaling pathway" and "MAPK

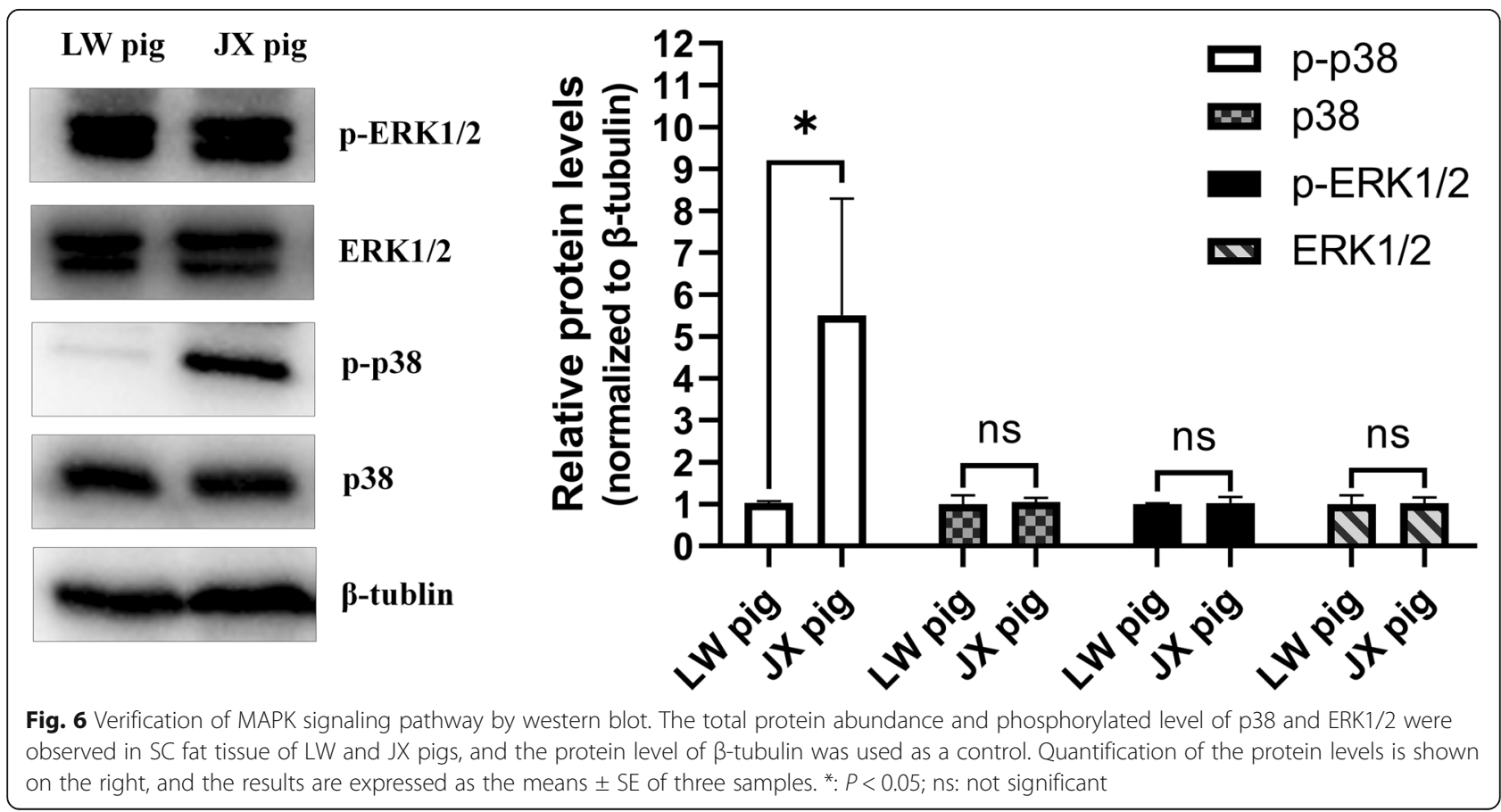


signaling pathway". The calcium signaling pathway can regulate lipolysis and the accumulation of adipose tissue by changing the concentration of calcium ions in adipocytes $[29,30]$. PI3Ks are a group of intracellular lipid kinases, which phosphorylate phosphatidylinositol and phosphoinositide to generate new intracellular second messengers [31]. These messengers in turn activate many intracellular signaling pathways and regulate various biological process in cells [32]. Plum et al.[33] reported that the PI3K signaling pathway is induced by leptin and participates in biological processes related to obesity. Insulin signaling via the PI3K/Akt axis plays an important role within adipocytes of obese patients, where the excess of lipids has to be properly stored in fat tissue [34]. The MAPK signaling pathway is common to several cell types, and it is involved in many biological processes, including cell proliferation, differentiation, development, and apoptosis [35]. The MAPK signaling pathway was among the top 20 most highly enriched pathways in KEGG analysis, and the GO annotation of DEGs uncovered the ERK1-ERK2 cascade, which indicates the activity of MAPK signaling pathway [36]. Several studies have shown that the MAPK pathway regulates the expression of adipogenic transcription factors during the adipogenesis [37]. The oncogenic form of Ras protein is a strong activator of the ERK pathway, and transfection of $3 \mathrm{~T} 3-\mathrm{L} 1$ preadipocyte cells with an activated Ras expression vector led to growth arrest and terminal adipocyte differentiation. The expression of the key adipogenic regulators $\mathrm{C} / \mathrm{EBP} \alpha, \beta$, and $\delta$, as well as PPAR $\gamma$ requires ERK activity, but the phosphorylation of PPARY caused by ERK inhibits adipocyte differentiation [38]. A study of the role of p38 MAPK signaling in regulating fat deposition in mice suggested that p38 MAPK could inhibit adipogenic differentiation by inhibiting the activity and expression of C/EBP $\beta$ and PPAR $\gamma$ during the whole process of lipogenesis in mice [39]. Owing to the opposing roles described for the MAPK pathway, one hypothesis states that the activation of MAPK can have different effects depending on the stage of differentiation. This hypothesis is in agreement with the fact that the differentiation process of $3 \mathrm{~T} 3-\mathrm{L} 1$ adipocytes requires a specific proliferative step (called mitotic clonal expansion, MCE) at the beginning [38, 40]. In addition, p38 MAPK activity was significantly higher in preadipocytes than in adipocytes [39], again suggesting that p38 MAPK activity has to be regulated in a timely manner during adipocyte differentiation. Therefore, the results of this study indicate that the MAPK signaling pathway may be important for mediating SC adipogenesis in JX pigs, and the difference between the two breeds may be related to mitogenic activity in preadipocytes regulated by the MAPK pathway. Considering that ERK and p38 are two main kinases in the MAPK pathway, and opposite results were observed for the regulatory functions of ERK and p38 during adipocyte differentiation $[38,41,42]$, we further compared the activation of p38/ ERK in SC adipose tissue of the two pig breeds. JX pigs showed a higher level of p38 phosphorylation in adipocytes than LW pigs. Previous research showed that the p38 MAPK signal could negatively regulate preadipocyte differentiation as well as SC and IM fat deposition in broiler chickens [43], as well as inhibit lipogenesis in 3T3-L1 adipocyte [44]. However, most studies performed in cell lines described a positive role of p38 in adipogenesis, and a decrease of $C / E B P \beta$ phosphorylation as well as PPAR $\gamma$ transactivation activity was observed when p38 activation was inhibited [38]. Moreover, mice lacking p38 $\alpha$ in adipose tissues displayed a lean phenotype, improved metabolism, and resistance to dietinduced obesity[45]. Thus, a precise understanding of the regulatory mechanism of these two pathways will be crucial for revealing the reason why JX pigs have a lower lean carcass weight ratio, and will be helpful for efficiently treating obesity.

Healthy adipose tissue is minimally infiltrated by immune cells, which serve as sentinels to detect invaders $[46,47]$. These cells also exert housekeeping functions that help maintain tissue integrity $[48,49]$. In this study, we discovered that many immune-related signaling pathways, such as the inflammatory response, adaptive immune response, tuberculosis, B cell receptor signaling pathway, phagosome and cytokine-cytokine receptor interaction, were enriched in GO and KEGG enrichment analysis. Thus, the obtained differential expression profiles of mRNAs and lncRNAs indicated that there are potential functions of cytokines and immune cells in the fat deposition and metabolism of pigs. Previous reports found that adipose tissue contains not only adipocytes but also fibroblasts, endothelial cells and a wide array of immune cells (adipose tissue macrophages, neutrophils, mast cells, eosinophils, $\mathrm{T}$ and $\mathrm{B}$ cells) that maintain tissue homeostasis [50]. The adipocyte expansion caused by a positive energy balance results in the expression of chemoattractant molecules and infiltration of inflammatory cells [51]. This difference of immune-related signaling pathways may be associated with the excessive SC fat deposition in JX pigs. However, the adipose tissue-derived adipokines are also crucial players in inflammation and immunity. For example, leptin has been shown to regulate both innate and adaptive immune responses, and its receptor is expressed throughout the immune system [52]. Proinflammatory cytokines, which promote lipolysis, have been traditionally considered to antagonize adipocyte differentiation. Nevertheless, transient low-level inflammation actually stimulates adipogenesis in animal models, and a transient inflammatory response is essential for healthy expansion of white 
adipose tissue through adipocyte progenitor recruitment [53]. Thus, there's a link between the immune response and fat metabolism, and it controls the homeostasis of adipose tissue. In addition to maintaining the structural integrity, these pathways can also regulate the endocrine functions of adipose tissues $[54,55]$. Consequently, our results provide evidence that $\mathrm{SC}$ adipogenesis in pigs is related to the immune landscape of porcine adipose tissues.

In the protein-protein interaction network of the identified DEGs, twelve hub genes were identified by the MCC topological algorithm. VCL encodes the cytoskeletal focal adhesion protein vinculin, and its depletion in mesenchymal stem cells (MSCs) promoted adipocyte differentiation on rigid substrates, as well as markedly increasing the mRNA expression of adipogenesis markers $P P A R \gamma 2$ and $a P 2$ [56]. It has been reported that various TLRs, such as NOD1, and NOD2, are expressed in adipocytes and adipose tissue of mouse and human origin. Moreover, the proinflammatory environment induced by the activation of TLR4 or TLR2 leads to the suppression of adipocyte differentiation [57]. Interferon regulatory factor 7 (IRF7) physically interacts with GSDMD and subsequently forms a complex to promote adipocyte pyroptosis [58]. The lysosomal protease cathepsin $\mathrm{Z}$ (CTSZ) is necessary for mitochondrial respiration in both mouse and human brown adipocytes, and it is markedly induced by cold [59]. In our study, a lower expression of these genes in JX pigs than in LW pigs was observed. SOCS3 has been reported to play a central role in metabolism as well as regulate the expression of SREBP1c, which is an important transcriptional factor of genes involved in lipid synthesis. Furthermore, SOCS3 gene variations are associated with disturbances of lipid metabolism [60]. The transcription factor FOS is a member of the AP-1 complex, and knockdown of FOS abolished the ongoing differentiation process [61]. Adipocyte-derived angiotensinogen (AGT) plays a role in both local adipose tissue development and in the endocrine system, and mice that overexpress $A G T$ in adipose tissue developed obesity with adipocyte hypertrophy, concurrent with insulin resistance and increased expression of lipogenic and pro-inflammatory makers [62]. It should be noted that other types of proteinases are also known to be involved in adipogenesis. Some matrix metalloproteases (MMPs) are expressed in adipose tissues, where they can act as a paracrine factor as well as secreted into the blood. Several groups showed that MMP2 and MMP9 possess adipogenesis-enhancing activity [63]. In the predicted protein-protein interaction network constructed in this study, MMP9 is located at a key central node. Our results show that SOCS3, FOS, $A G T$ and MMP9 have higher expression in JX pigs. Taken together, these regulatory relationships may partly explain the mechanism of porcine SC deposition.
The biological function of lncRNAs is usually mediated by their target genes. Thus, the target genes of the identified DELs were predicted and subjected to enrichment analysis. Our results indicated that the lncRNAs LTCONS_00049888, LTCONS_00077321, LTCONS_ 00060219, LTCONS_00006788, LTCONS_00055593, LTCONS_00029009, LTCONS_00031822 and XR 002341580.1 regulate AKAP9, CDC25B, LPAR1, Nfe2l1, UVRAG, CAPZB and WEE1, respectively. AKAP9 regulates activation-induced retention of $\mathrm{T}$ lymphocytes at sites of inflammation and is related to the activation of the MAPK pathway in thyroid cancer [64]. In addition, CDC25B, LPAR1, UVRAG and CAPZB regulate cell growth, morphology and differentiation. It is worth noting that LTCONS_00055593 may play critical roles in fat metabolism and deposition by regulating its target gene $N f e 2 l 1$, which affects the plasticity of adipose tissue [65]. These results indicate that the DELs might participate in cell proliferation or differentiation, and their function in SC adipogenesis should be confirmed by further analysis in future studies.

The DEGs and DELs identified in this study possibly reflect differences of fat deposition or deeper genetic differences between the two breeds. According to GO and KEGG analysis, several pathways related to lipid metabolism were enriched, including"positive regulation of ERK1 and ERK2 cascade", "Calcium signaling pathway", "PI3K-Akt signaling pathway", "Osteoclast differentiation", and "MAPK signaling pathway", which may account for the difference of fat deposition between the two breeds. Western blot analysis confirmed the results of enrichment. Our findings expand the knowledge of the regulatory network in porcine SC adipocytes, which is necessary for the rational breeding of pigs for improved fat-related traits.

\section{Conclusions}

In summary, a comparative transcriptome analysis of porcine SC adipocytes between JX and LW pigs was conducted. A large number of DEGs and DELs were identified. Elevated activity of the MAPK/p38 pathway was detected in the SC fat of JX pigs. Taken together, the results may help explain the excessive fat deposition of JX pigs and offer a clue for genetic improvement of Chinese domestic pig breeds.

\section{Methods}

\section{Experimental animals}

All experimental procedures involving animals were performed in accordance with the guidelines of the Animal Care and Use Committee at the Jiaxing University. The experimental animals used here included 3 male JX pigs and 3 male LW pigs, which were 3-day-old and provided by Zhejiang Qinglian Food Co., Ltd. (Jiaxing, Zhejiang 
Province, China). The piglets were raised under the same feeding and environmental conditions. All piglets were sacrificed using a $\mathrm{CO}_{2}$ euthanasia box, after which the $\mathrm{SC}$ adipose tissues were collected for the primary culture of SC adipocytes.

\section{Preadipocyte culture and differentiation}

Porcine SC adipose tissue isolated from male piglet was washed three times with serum-free DMEM/F12 medium. Then, tissues were aseptically cut into pieces and incubated with $1 \mathrm{mg} / \mathrm{mL}$ type I collagenase (Invitrogen, Carlsbad, CA, USA) at $37^{\circ} \mathrm{C}$ for an hour. The digestion solution was filtrated through a $200 \mu \mathrm{m}$ nylon mesh, after which the preadipocytes were collected by centrifuged at $1000 \mathrm{rpm}$ for $10 \mathrm{~min}$, and cultured in DMEM/ F12 medium (HyClone, USA) containing 10\% fetal bovine serum (FBS; Gibco, USB) and $1 \%$ penicillinstreptomycin at $37^{\circ} \mathrm{C}$ in a humidified atmosphere comprising 5\% $\mathrm{CO}_{2}$. After the preadipocytes reached confluence (designated as experimental day 0), the differentiation cocktail comprising DMEM/F12 supplemented with $10 \%$ FBS, $0.5 \mathrm{mM}$ isobutyl methylxanthine (IBMX; Sigma, USA), $0.5 \mathrm{mM}$ dexamethasone (Sigma, USA), and $20 \mathrm{nM}$ insulin (Sigma, USA) was added to induce cell differentiation for 2 days, after which the cells were maintained in DMEM/F12 medium supplemented with $10 \%$ FBS and $20 \mathrm{nM}$ insulin for an additional 8 days.

\section{RNA isolations and Illumina sequencing}

Differentiated SC adipocytes from three biological replicates were subjected for RNA isolation using Trizol reagent (Invitrogen, USA). The RNA quality and concentration were measured using a NanoDrop One (Thermo Fisher Scientific, USA) and agarose gel electrophoresis. The RNA was stored at $-80^{\circ} \mathrm{C}$ until further use. Ribosomal RNA from each sample was removed using the Ribo-Zero ${ }^{\text {Tw }}$ rRNA Removal Kit (Epicentre, USA), after which the purified RNA was fragmented and reversely transcribed to synthesize the first-strand cDNA using the TruSeq ${ }^{\bullet}$ First-Strand kit (Illumina, USA). The double-stranded cDNA (ds-cDNA) was synthesized in a reaction mixture comprising buffer, dNTPs, RNase $\mathrm{H}$ and DNA polymerase I, and the end of ds-cDNA was ligated with an 'A' base and sequencing linker. The entire library was completed by performing amplification of ligation products, and the paired-end sequencing reads generated by the Illumina 2000 platform had a length of $2 \times 90 \mathrm{bp}$.

\section{Reference genome mapping and gene quantification}

After removing low-quality reads from the raw reads using SOAP, the remaining sequences were aligned to the reference genome Sscrofa 11.1 using HISAT software. The resulting alignment data were then fed into StringTie for transcriptome assembly, and Cufflinks was used to map the sequencing transcripts to reference transcripts. Subsequently, the abundance of transcripts was determined using the Fragments Per Kilobase of exon per Million fragments mapped (FPKM) method.

\section{LncRNA identification}

The criteria for candidate IncRNAs identification were as follows: Firstly, transcripts shorter than 200 nt or less than two exons were filtered out; Secondly, the tools encoding potential calculator (CPC), coding-non-coding index (CNCI), txCdsPredict and protein folding domain database (Pfam) were used for lncRNA screening. The lncRNAs that appeared as hits in at least three of the four software tools were included in the final result. To identify the known lncRNAs, lncRNA candidates were aligned to the ALDB database (A Domestic Animal Long Noncoding RNA Database) with the following settings: identity $(100 \%)$, mismatch $(0)$, E-value $(<1 \mathrm{e}-10)$ and gap opening (0).

\section{Differential expression analysis}

The RSEM [66] software package was used to quantify gene abundance, and StringTie [67] was used to calculate FPKMs of both lncRNAs and coding genes in each sample. The differential expression analysis of genes with average FPKM of $\geq 1.0$ in a pig breed was performed using the DESeq2 $\mathrm{R}$ package [68]. Genes and lncRNAs with an adjusted $P$ value $<0.05$ and absolute fold change $\geq 2$ were considered to be differentially expressed. A volcano plot of differentially expressed genes was rendered using the ggpubr $\mathrm{R}$ package, and heat maps were plotted using MeV 4.9 software.

\section{LncRNA target gene prediction}

LncRNAs can regulate target genes by acting in cis or in trans, and genes with similar expression patterns might exhibit high correlation in biological functions. Accordingly, the target genes of IncRNAs were predicted as follows. For the significance of the proximity of coregulated IncRNA and mRNAs, Spearman and Pearson correlation coefficients were calculated based on the expression values of each lncRNA and mRNA, and coexpressed lncRNA-mRNA pairs with correlation coefficients $\geq 0.6$ were selected. When these target genes were located $10 \mathrm{~kb}$ upstream or $20 \mathrm{~kb}$ downstream of the lncRNAs, they were considered as cis target genes. Beyond this location, RNAplex [69] was applied to select trans-acting target genes according to the interaction between IncRNA and mRNA sequences with binding energy $<-30$. 
Enrichment analyses and construction of the proteinprotein interaction network

GO annotation and KEGG pathway enrichment analysis were performed for DEGs and lncRNA target genes to explore the main biological functions of the differentially expressed mRNA and lncRNAs. The statistical enrichment of DEGs was analyzed using DAVID 6.8. The STRI NG website was used to construct a protein-protein interaction network, and the Cytoscape software was used to visualize it.

\section{Quantitative real-time RT-PCR}

Gene and lncRNA specific primers were designed using the Primer-BLAST tool, and the amplification efficiency of primers was confirmed by general PCR (Additional file 1: Supplementary Table 5). Approximately $0.5 \mu \mathrm{g}$ of each RNA sample was used to synthesize cDNA templates using the HiFiScript gDNA Removal cDNA kit (CWbiotech, China). A QuantStudio3 Real-time PCR Instrument (Thermo Fisher Scientific, USA) was used for the qRT-PCR assay, and the reaction system was set up according to the manufacturer's instructions of the $2 \times$ Plus SYBR real-time PCR kit (CWbiotech). The temperature program encompassed an initial denaturation step at $94^{\circ} \mathrm{C}$ for $3 \mathrm{~min}$, followed by 40 cycles of $95^{\circ} \mathrm{C}$ for $15 \mathrm{~s}, 60^{\circ} \mathrm{C}$ for $15 \mathrm{~s}$, and $72{ }^{\circ} \mathrm{C}$ for 30s. The housekeeping gene $\beta$-actin was used as the control for normalization, and the experiments were performed in triplicate. The $2^{-\Delta \Delta C T}$ method was used to calculate the relative gene expression levels.

\section{Western blot analysis}

RIPA buffer (Beyotime, Shanghai, China) supplemented with protease inhibitor (Pierce, Bradenton, Florida, USA) was used to extract the total proteins from SC fat tissue of three JX and three LW pigs (3 days old). The lysates were centrifuged at $12000 \mathrm{rpm}$ for $10 \mathrm{~min}$, and the supernatant was boiled in sodium dodecyl sulfate (SDS) loading buffer (Beyotime, Shanghai, China). After separation on a $12 \%$ acrylamide SDS-PAGE gel, the protein bands were transferred onto a PVDF membrane (Millipore, Massachusetts, USA). The membrane was incubated with different primary antibodies, against p38, p-p38, pERK1/2 and ppERK1/2, respectively, which were purchased from cell signal Technology. A Bio-Rad ChemiDoc XRS+ image analyzer system was used to photograph the blots.

\section{Statistical analysis}

All data were presented as the means \pm standard error (SE), and the statistical analysis of the qRT-PCR assay was implemented using GraphPad Prism 8. The statistical significance of differences between JX and LW pigs was assessed using Student's $t$-test, with $P<0.05$ as the threshold.

\section{Supplementary Information}

The online version contains supplementary material available at https://doi. org/10.1186/s12864-020-07361-9.

Additional file 1.

Additional file 2.

Additional file 3.

\section{Abbreviations}

SC: Subcutaneous; IM: Intramuscular; IncRNAs: long noncoding RNAs; JX pig: Jiaxing Black pig; LW pig: Large White pig; DEGs: Differentially expressed genes; DELs: Differentially expressed IncRNAs; GO: Gene Ontology; KEGG: Kyoto Encyclopedia of Genes and Genomes; MCC: Maximal Clique Centrality; MMPs: matrix metalloproteases

\section{Acknowledgements}

The authors are grateful to Zhejiang Qinglian Food Co., Ltd., Jiaxing Dayunhe Ecological Animal Husbandry Co., Ltd. and Zhejiang Huatong meat products Co., Ltd. for providing experimental animals.

\section{Authors' contributions}

DZ designed the experiments and wrote the paper, and provided the experimental funding. WW designed the experiments and performed cell culture. DZ, XH, CZ and KX performed, analyzed and interpreted the study. $J Z$ designed the study, revised the paper, and provided the experimental funding. All authors analyzed the results and approved the final version of the manuscript.

\section{Funding}

This work was supported by Zhejiang Natural Science Foundation (LY20C170003), Initial Scientific Research Fund of Young Teachers in Jiaxing University (No.70518061) and General Research Project of Zhejiang Provincial Department of Education (No. Y201942267).

\section{Availability of data and materials}

The data sets supporting the results of this article are included within the manuscript and its additional files. The raw datasets generated in this study were deposited in the Sequence Read Archive under the accession number PRJNA631903.

\section{Ethics approval and consent to participate}

Jiaxing University Animal Care Committee provided official ethics board approval for this study. Tissues used in this study were collected from pigs maintained and used according to the approved protocols.

\section{Consent for publication}

Not applicable.

\section{Competing interests}

The authors declare that they have no competing interests.

\section{Author details}

${ }^{1}$ College of Biological, Chemical Science and Engineering, Jiaxing University, Jiaxing 314001, China. ${ }^{2}$ College of Agronomy and Biotechnology, Hebei Normal University of Science and Technology, Qin Huangdao 066000, Hebei, China.

Received: 26 March 2020 Accepted: 30 December 2020 Published online: 19 January 2021

\section{References}

1. Wu WJ, Zhang DW, Yin YJ, Ji M, Xu K, Huang X, Peng YJ, Zhang J. Comprehensive transcriptomic view of the role of the LGALS12 gene in porcine subcutaneous and intramuscular adipocytes. BMC Genomics. 2019; 20(1):509.

2. Li MZ, Zhu L, Li XX, Shuai SR, Teng XK, Xiao HS, Li Q, Chen L, Guo YJ, Wang $J Y$. Expression profiling analysis for genes related to meat quality and carcass traits during postnatal development of backfat in two pig breeds. Sci China C Life Sci. 2008;51 (8):718-33. 
3. Newcom DW, Baas TJ, Schwab CR, Stalder KJ. Genetic and phenotypic relationships between individual subcutaneous backfat layers and percentage of longissimus intramuscular fat in Duroc swine. J Anim Sci. 2005;83(2):316-23.

4. Cui JX, Zeng YQ, Wang $H$, Chen W, Du JF, Chen QM, Hu YX, Yang L. The effects of DGAT1 and DGAT2 mRNA expression on fat deposition in fatty and lean breeds of pig. Livest Sci. 2011;140(1-3):292-6.

5. Yuan ZQ, Song DG, Wang YZ. The novel gene PFAM134B positively regulates fat deposition in the subcutaneous fat of Sus scrofa. Biochem Biophys Res Commun. 2014;454(4):554-9.

6. Yan WJ, Li XM, Jiang YX. Fatty Acids in Intramuscular and Subcutaneous Fat of Jinhua Ham. Food Ferment Ind. 2005;2:124-6.

7. Liu X, Liu KQ, Shan BS, Wei SJ, Li DF, Han HY, Wei W, Chen J, Liu HL, Zhang LF. A genome-wide landscape of mRNAs, IncRNAs, and circRNAs during subcutaneous adipogenesis in pigs. J Anim Sci Biotechnol. 2018;9:76.

8. Miao ZG, Wang S, Zhang JZ, Wei PP, Guo LP, Liu DY, Wang YM, Shi MY. Identification and comparison of long non-conding RNA in Jinhua and Landrace pigs. Biochem Biophys Res Commun. 2018;506(3):765-71.

9. Chen H, Huang M, Yang B, Wu ZP, Deng Z, Hou Y, Ren J, Huang LS. Introgression of Eastern Chinese and Southern Chinese haplotypes contributes to the improvement of fertility and immunity in European modern pigs. Gigascience. 2020;9(3):giaa014.

10. Huang WL, Zhang XX, Li A, Xie LL, Miao XY. Genome-wide analysis of mRNAs and IncRNAs of intramuscular fat related to lipid metabolism in two pig breeds. Cell Physiol Biochem. 2018;50(6):2406-22.

11. Crujeiras AB, Díaz-Lagares A, Carreira MC, Amil M, Casanueva FF. Oxidative stress associated to dysfunctional adipose tissue: a potential link between obesity, type 2 diabetes mellitus and breast cancer. Free Radic Res. 2013; 47(4):243-56.

12. Medina-Remón A, Kirwan R, Lamuela-Raventós RM, Estruch R. Dietary patterns and the risk of obesity, type 2 diabetes mellitus, cardiovascular diseases, asthma, and neurodegenerative diseases. Crit Rev Food Sci Nutr. 2018;58(2):262-96.

13. Kuzmuk KN, Schook LB. Pigs as a model for biomedical sciences. Genet Pig. 2011;2:426-44

14. Gün $G$, Kues WA. Current progress of genetically engineered pig models for biomedical research. Biores Open Access. 2014;3(6):255-64.

15. Zhang GH, Lu JX, Chen Y, Zhao YQ, Guo PH, Yang JT, Zang RX. Comparison of the adipogenesis in intramuscular and subcutaneous adipocytes from Bamei and Landrace pigs. Biochem Cell Biol. 2014;92(4):259-67.

16. Hou XH, Yang YL, Zhu SY, Hua CJ, Zhou R, Mu YL, Tang ZL, Li K Comparison of skeletal muscle miRNA and mRNA profiles among three pig breeds. Mol Gen Genomics. 2016;291(2):559-73.

17. de Almeida AM, Bendixen E. Pig proteomics: a review of a species in the crossroad between biomedical and food sciences. J Proteome. 2012;75(14): 4296-314.

18. Murgiano L, D'Alessandro A, Egidi MG, Crisa A, Prosperini G, Timperio AM, Valentini A, Zolla L. Proteomics and transcriptomics investigation on longissimus muscles in Large White and Casertana pig breeds. J Proteome Res. 2010;9(12):6450-66.

19. Zhang J, He Q, Liu QY, Guo W, Deng XM, Zhang WW, Hu XX, Li N. Differential gene expression profile in pig adipose tissue treated with/ without clenbuterol. BMC Genomics. 2007;8:433.

20. Ji M, Xu K, Zhang DW, Chen TT, Shen LC, Wu WW, Zhang J. Adipose-TissueSpecific Expression of Pig ApoR Protects Mice from Diet-Induced Obesity. J Agric Food Chem. 2020;68(7):2256-62.

21. Wu WW, Ji M, Xu K, Zhang DW, Yin YJ, Huang X, Peng YJ, Zhang J. Knockdown of CTRP6 reduces the deposition of intramuscular and subcutaneous fat in pigs via different signaling pathways. Biochim Biophys Acta Mol Cell Biol Lipids. 2020;1865(8):158729.

22. Schmitz SU, Grote P, Herrmann BG. Mechanisms of long noncoding RNA function in development and disease. Cell Mol Life Sci. 2016;73(13):2491-509.

23. van Solingen C, Scacalossi KR, Moore KJ. Long noncoding RNAs in lipid metabolism. Curr Opin Lipidol. 2018;29(3):224.

24. Zhang W, Song QQ, Wu F, Zhang JZ, Xu MS, Li HH, Han ZJ, Gao HX, Xu NY. Evaluation of the four breeds in synthetic line of Jiaxing Black Pigs and Berkshire for meat quality traits, carcass characteristics, and flavor substances. Anim Sci J. 2019;90(4):574-82.

25. Cohen P, Levy JD, Zhang Y, Frontini A, Kolodin DP, Svensson KJ, Lo JC, Zeng X, Ye L, Khandekar MJ. Ablation of PRDM16 and beige adipose causes metabolic dysfunction and a subcutaneous to visceral fat switch. Cell. 2014; 156(1-2):304-16.
26. Poulos SP, Hausman DB, Hausman GJ. The development and endocrine functions of adipose tissue. Mol Cell Endocrinol. 2010;323(1):20-34.

27. He YH, Zhang HQ, Teng JH, Huang LN, Li Y, Sun CH. Involvement of calcium-sensing receptor in inhibition of lipolysis through intracellular CAMP and calcium pathways in human adipocytes. Biochem Biophys Res Commun. 2011:404(1):393-9.

28. Xue B, Greenberg AG, Kraemer FB, Zemel MB. Mechanism of intracellular calcium ([Ca2+] i) inhibition of lipolysis in human adipocytes. FASEB J. 2001; 15(13):2527-9.

29. Li H, Marshall AJ. Phosphatidylinositol $(3,4)$ bisphosphate-specific phosphatases and effector proteins: a distinct branch of PI3K signaling. Cell Signal. 2015;27(9):1789-98.

30. Manning BD, Tee AR, Logsdon MN, Blenis J, Cantley LC. Identification of the tuberous sclerosis complex-2 tumor suppressor gene product tuberin as a target of the phosphoinositide 3-kinase/akt pathway. Mol Cell. 2002;10(1): $151-62$.

31. Plum L, Rother E, Münzberg H, Wunderlich FT, Morgan DA, Hampel B, Shanabrough M, Janoschek R, Könner AC, Alber J, Suzuki A, Krone W, Horvath TL, Rahmouni K, Brüning JC. Enhanced leptin-stimulated Pi3k activation in the CNS promotes white adipose tissue transdifferentiation. Cell Metab. 2007:6(6):431-45.

32. Sharma BR, Kim HJ, Rhyu DY. Caulerpa lentillifera extract ameliorates insulin resistance and regulates glucose metabolism in C57BL/KsJ-db/db mice via PI3KJAKT signaling pathway in myocytes. J Transl Med. 2015;13(1):62.

33. Sun Y, Liu W-Z, Liu T, Feng X, Yang N, Zhou H-F. Signaling pathway of MAPK/ERK in cell proliferation, differentiation, migration, senescence and apoptosis. J Recept Signal Transduct Res. 2015;35(6):600-4.

34. Capolongo G, Suzumoto Y, D'Acierno M, Simeoni M, Capasso G, Zacchia M. ERK1, 2 Signalling Pathway along the Nephron and Its Role in Acid-base and Electrolytes Balance. Int J Mol Sci. 2019;20(17):4153.

35. Kim JK, So H, Youn MJ, Kim HJ, Kim Y, Park C, Kim SJ, Ha YA, Chai KY, Kim SM, Kim KY, Park R. Hibiscus sabdariffa L. Hibiscus sabdariffa L. water extract inhibits the adipocyte differentiation through the PI3-K and MAPK pathway. J Ethnopharmacol. 2007;114(2):260-7.

36. Bost F, Aouadi M, Caron L, Binetruy B. The role of MAPKs in adipocyte differentiation and obesity. Biochimie. 2005;87(1):51-6.

37. Aouadi M, Laurent K, Prot M, Le Marchand-Brustel Y, Binetruy B, Bost F. Inhibition of p38MAPK increases adipogenesis from embryonic to adult stages. Diabetes. 2006;55(2):281-9.

38. Tang QQ, Otto TC, Lane MD. Mitotic clonal expansion: a synchronous process required for adipogenesis. Proc Natl Acad Sci U S A. 2003; 100(1):44-9.

39. Cao DD, Ma FF, Ouyang SR, Liu Z, Li YY, Wu JX. Effects of macrophages and CXCR2 on adipogenic differentiation of bone marrow mesenchymal stem cells. J Cell Physiol. 2019;234(6):9475-85.

40. Deng W, Chen HD, Su HJ, Wu XH, Xie ZY, Wu YF, Shen HY. IL6 Receptor Facilitates Adipogenesis Differentiation of Human Mesenchymal Stem Cells through Activating P38 Pathway. Int J Stem Cells. 2020;13(1):142-50.

41. Yan J, Gan L, Qi RL, Sun C. Adiponectin decreases lipids deposition by p38 MAPKJATF2 signaling pathway in muscle of broilers. Mol Biol Rep. 2013; 40(12):7017-25.

42. Feng M, Tian L, Gan L, Liu ZJ, Sun C. Mark4 promotes adipogenesis and triggers apoptosis in 3T3-L1 adipocytes by activating JNK1 and inhibiting p38MAPK pathways. Biol Cell. 2014;106(9):294-307.

43. Zhang S, Cao H, Li Y, Jing Y, Liu S, Ye C, Wang H, Yu S, Peng C, Hui L, Wang YC, Zhang H, Guo F, Zhai Q, Wang H, Huang R, Zhang L, Jiang J, Liu W, Ying $\mathrm{H}$. Metabolic benefits of inhibition of p38alpha in white adipose tissue in obesity. PLoS Biol. 2018;16(5):e2004225.

44. Lolmède K, Duffaut C, Zakaroff-Girard A, Bouloumié A. Immune cells in adipose tissue: key players in metabolic disorders. Diabetes Metab. 2011; 37(4):283-90.

45. Mraz M, Haluzik M. The role of adipose tissue immune cells in obesity and low-grade inflammation. J Endocrinol. 2014;222(3):R113-27.

46. Majdoubi A, Kishta OA, Thibodeau J. Role of antigen presentation in the production of pro-inflammatory cytokines in obese adipose tissue. Cytokine. 2016:82:112-21.

47. Guzik TJ, Skiba DS, Touyz RM, Harrison DG. The role of infiltrating immune cells in dysfunctional adipose tissue. Cardiovasc Res. 2017:113(9):1009-23.

48. Huh JY, Park YJ, Ham M, Kim JB. Crosstalk between adipocytes and immune cells in adipose tissue inflammation and metabolic dysregulation in obesity. Mol Cell. 2014;37(5):365-71. 
49. Vieira-Potter VJ. Inflammation and macrophage modulation in adipose tissues. Cell Microbiol. 2014;16(10):1484-92.

50. Francisco V, Pino J, Campos-Cabaleiro V, Ruiz-Fernandez C, Mera A, Gonzalez-Gay MA, Gomez R, Gualillo O. Obesity, Fat Mass and Immune System: Role for Leptin. Front Physiol. 2018;9:640.

51. Sun K, Gao Z, Kolonin MG. Transient inflammatory signaling promotes beige adipogenesis. Sci Signal. 2018;11(527):eaat3192.

52. Lumeng CN. Innate immune activation in obesity. Mol Asp Med. 2013;34(1): $12-29$.

53. Joffe $\mathrm{YT}$, Collins $\mathrm{M}$, Goedecke JH. The relationship between dietary fatty acids and inflammatory genes on the obese phenotype and serum lipids. Nutrients. 2013;5(5):1672-705.

54. Kuroda M, Wada H, Kimura Y, Ueda K, Kioka N. Vinculin promotes nuclear localization of TAZ to inhibit ECM stiffness-dependent differentiation into adipocytes. J Cell Sci. 2017;130(5):989-1002.

55. S Purohit J, Hu P, Burke SJ, Collier JJ, Chen J, Zhao L. The effects of NOD activation on adipocyte differentiation. Obesity (Silver Spring). 2013;21(4): $737-47$

56. Liu ZJ, Gan L, Xu YT, Luo D, Ren Q, Wu S, Sun C. Melatonin alleviates inflammasome-induced pyroptosis through inhibiting NF-KB/GSDMD signal in mice adipose tissue. J Pineal Res. 2017;63(1):e12414.

57. Shen Y, Su Y, Silva FJ, Weller AH, Sostre-Colón J, Titchenell PM, Steger DJ, Seale P, Soccio RE. Shared PPARa/Y Target Genes Regulate Brown Adipocyte Thermogenic Function. Cell Rep. 2020;30(9):3079-91.

58. Xu Y, Wu Y, Xiong Y, Tao J, Pan T, Tan S, Gao G, Chen Y, Abbas N, Getachew A, Zhuang Y, You K, Yang F, Li YX. Ascorbate protects liver from metabolic disorder through inhibition of lipogenesis and suppressor of cytokine signaling 3 (SOCS3). Nutr Metab (Lond). 2020;17:17.

59. Knebel B, Kotzka J, Lehr S, Hartwig S, Avci H, Jacob S, Nitzgen U, Schiller M, März W, Hoffmann MM, Seemanova E, Haas J, Muller-Wieland D. A mutation in the c-fos gene associated with congenital generalized lipodystrophy. Orphanet J Rare Dis. 2013;8:119.

60. Carroll WX, Kalupahana NS, Booker SL, Siriwardhana N, Lemieux M, Saxton AM, Moustaid-Moussa N. Angiotensinogen gene silencing reduces markers of lipid accumulation and inflammation in cultured adipocytes. Front Endocrinol (Lausanne). 2013;4:10.

61. Van Hul M, Lijnen HR. Matrix metalloproteinase inhibition impairs murine adipose tissue development independently of leptin. Endocr J. 2011;58(2): $101-7$.

62. Ciampi R, Knauf JA, Kerler R, Gandhi M, Zhu Z, Nikiforova MN, Rabes HM, Fagin JA, Nikiforov YE. Oncogenic AKAP9-BRAF fusion is a novel mechanism of MAPK pathway activation in thyroid cancer. J Clin Invest. 2005;115(1):94-101.

63. Hou YY, Liu ZY, Zuo Z, Gao TC, Fu JQ, Wang HH, Xu YY, Liu DX, Yamamoto M, Zhu BB, Zhang YG, Andersen ME, Zhang Q, Pi JB. Adipocyte-specific deficiency of Nfe2l1 disrupts plasticity of white adipose tissues and metabolic homeostasis in mice. Biochem Biophys Res Commun. 2018; 503(1):264-70

64. Li B, Dewey CN. RSEM: accurate transcript quantification from RNA-Seq data with or without a reference genome. BMC Bioinformatics. 2011:12:323.

65. Pertea M, Pertea GM, Antonescu CM, Chang TC, Mendell JT, Salzberg SL. Stringtie enables improved reconstruction of a transcriptome from RNA-seq reads. Nat Biotechnol. 2015;33(3):290-5.

66. Wang LK, Feng ZX, Wang X, Wang XW, Zhang XG. DEGseq: an R package for identifying differentially expressed genes from RNA-seq data. Bioinformatics. 2010;26(1):136-8.

67. Tafer H, Amman F, Eggenhofer F, Stadler PF, Hofacker IL. Fast accessibilitybased prediction of RNA-RNA interactions. Bioinformatics. 2011;27(14):1934-40.

68. Liu X, Gong JF, Wang LG, Hou XH, Gao HM, Yan H, Zhao FP, Zhang LC, Wang LX. Genome-Wide Profiling of the Microrna Transcriptome Regulatory Network to Identify Putative Candidate Genes Associated with Backfat Deposition in Pigs. Animals (Basel). 2019;9(6):313.

69. Li HY, Xi QY, Xiong YY, Liu XL, Cheng X, Shu G, Wang SB, Wang LN, Gao P, Zhu XT, Jiang QY, Yuan L, Zhang YL. Identification and comparison of microRNAs from skeletal muscle and adipose tissues from two porcine breeds. Anim Genet. 2012;43(6):704-13.

\section{Publisher's Note}

Springer Nature remains neutral with regard to jurisdictional claims in published maps and institutional affiliations.

Ready to submit your research? Choose BMC and benefit from:

- fast, convenient online submission

- thorough peer review by experienced researchers in your field

- rapid publication on acceptance

- support for research data, including large and complex data types

- gold Open Access which fosters wider collaboration and increased citations

- maximum visibility for your research: over $100 \mathrm{M}$ website views per year

At BMC, research is always in progress.

Learn more biomedcentral.com/submissions 